Article

Subscriber access provided by King Abdullah University of Science and Technology Library

\title{
Treating sub-valence correlation effects in domain based pair natural orbital coupled cluster calculations: an out-of-the-box approach
}

Giovanni Bistoni, Christoph Riplinger, Yury Minenkov, Luigi Cavallo, Alexander A. Auer, and Frank Neese

J. Chem. Theory Comput., Just Accepted Manuscript • DOI: 10.1021/acs.jctc.7b00352 • Publication Date (Web): 12 Jun 2017

Downloaded from http://pubs.acs.org on June 18, 2017

\section{Just Accepted}

"Just Accepted" manuscripts have been peer-reviewed and accepted for publication. They are posted online prior to technical editing, formatting for publication and author proofing. The American Chemical Society provides "Just Accepted" as a free service to the research community to expedite the dissemination of scientific material as soon as possible after acceptance. "Just Accepted" manuscripts appear in full in PDF format accompanied by an HTML abstract. "Just Accepted" manuscripts have been fully peer reviewed, but should not be considered the official version of record. They are accessible to all readers and citable by the Digital Object Identifier (DOI®). "Just Accepted" is an optional service offered to authors. Therefore, the "Just Accepted" Web site may not include all articles that will be published in the journal. After a manuscript is technically edited and formatted, it will be removed from the "Just Accepted" Web site and published as an ASAP article. Note that technical editing may introduce minor changes to the manuscript text and/or graphics which could affect content, and all legal disclaimers and ethical guidelines that apply to the journal pertain. ACS cannot be held responsible for errors or consequences arising from the use of information contained in these "Just Accepted" manuscripts. 


\section{Treating sub-valence correlation effects in domain}

\section{based pair natural orbital coupled cluster}

\section{calculations: an out-of-the-box approach.}

Giovanni Bistoni, ${ }^{1}$ Christoph Riplinger, ${ }^{1}$ Yury Minenkov, ${ }^{2}$ Luigi Cavallo, ${ }^{2}$ Alexander A. Auer $^{1}$ and Frank Neese ${ }^{1 *}$

1) Department of Molecular Theory and Spectroscopy, Max Planck Institute for Chemical Energy Conversion, Stiftstr. 34-36, D-45470 Mülheim an der Ruhr, Germany. E-Mail: Frank.Neese@cec.mpg.de

2) Physical Sciences and Engineering Division, King Abdullah University of Science and Technology, KAUST Catalysis Center, Thuwal 23955-6900, Saudi Arabia. E-mail:

Yury.Minenkov@kaust.edu.sa, Luigi.Cavallo@kaust.edu.sa

ABSTRACT. The validity of the main approximations used in canonical and domain based pair natural orbital coupled cluster methods $(\operatorname{CCSD}(\mathrm{T})$ and $\operatorname{DLPNO}-\mathrm{CCSD}(\mathrm{T})$, respectively) in standard chemical applications is discussed. In particular, we investigate the dependence of the results on the number of electrons included in the correlation treatment in frozen-core (FC) calculations and on the main threshold governing the accuracy of DLPNO all-electron (AE) calculations. Initially, scalar relativistic orbital energies for the ground state of the atoms from Li to $\mathrm{Rn}$ in the periodic table are calculated. An energy criterion is used for determining the orbitals that can be excluded from the correlation treatment in FC coupled cluster calculations without significant loss of accuracy. The heterolytic dissociation energy (HDE) of a series of metal compounds ( $\mathrm{LiF}, \mathrm{NaF}, \mathrm{AlF}_{3}, \mathrm{CaF}_{2}, \mathrm{CuF}, \mathrm{GaF}_{3}, \mathrm{YF}_{3}, \mathrm{AgF}, \mathrm{InF}_{3}, \mathrm{HfF}_{4}$ and $\mathrm{AuF})$ is calculated at the canonical $\operatorname{CCSD}(\mathrm{T})$ level, and the dependence of the results on the number of correlated electrons is investigated. Although for many of the studied reactions sub-valence correlation effects contribute significantly to the HDE, the use of an energy 
criterion permits a conservative definition of the size of the core, allowing FC calculations to be performed in a black-box fashion while retaining chemical accuracy. A comparison of the CCSD and the DLPNO-CCSD methods in describing the core-core, core-valence and valence-valence components of the correlation energy is given. It is found that more conservative thresholds must be used for electron pairs containing at least one core electron in order to achieve high accuracy in AE DLPNO-CCSD calculations relative to FC calculations. With the new settings, the DLPNO-CCSD method reproduces canonical CCSD results in both $\mathrm{AE}$ and FC calculations with the same accuracy.

\section{Introduction}

In modern electronic structure theory, the hierarchy of coupled cluster (CC) ${ }^{1}$ methods stands out for being particularly accurate, robust and size-extensive. At the complete basis set limit, coupled cluster with single-, double-, and perturbatively included triple excitations CCSD(T) can be used in a black-box fashion for calculating thermochemical quantities with chemical accuracy $^{2-4}$ provided that the effect of the static correlation is small enough. For these reasons, $\operatorname{CCSD}(\mathrm{T})$ has been called the "gold standard" of computational chemistry and is often used as an alternative to experimental determinations when the accurate calculations of properties and reaction energies is the goal.

In its canonical implementation, the $\operatorname{CCSD}(\mathrm{T})$ method scales with $\mathrm{N}^{7}$ with the system size and can only be used for calculating energies of relatively small systems on commonly available hardware. In order to extend its applicability, a wide range of approximated methods, exploiting the locality of the electron correlation, have been developed. ${ }^{5-13}$ In this context, some of us recently contributed with the development of the domain based pair natural orbital coupled cluster DLPNO-CCSD(T) method, ${ }^{14-22}$ which relies on the concept of pair natural orbitals (PNOs). In its most recent, fully-linear scaling implementation, ${ }^{22}$ the 
DLPNO-CCSD(T) method typically recovers more than $99.9 \%$ of the correlation energy with respect to its canonical counterpart and can routinely be applied to systems for which single point DFT calculations are feasible. ${ }^{22-24}$

A further reduction of the computational cost of coupled cluster calculations can be obtained by means of the frozen-core (FC) approximation, which reduces the computational cost by including only valence orbitals in the correlation treatment. The idea behind the FC approximation is that dynamic correlation from core orbitals cancels out in the calculation of relative energies and, in fact, the FC approximation has already proven its accuracy in a wide range of situations. However, it is well known that sub-valence correlation effects play an important role in many cases, leading to large errors if only valence orbitals are included in the correlation treatment. ${ }^{25-36}$ A prototypical example is the case of the $4 \mathrm{f}$ orbitals of $\mathrm{Hf}$. Although formally considered as core and included in the widely used relativistic StuttgartDresden effective core potential, ${ }^{37}$ it has been shown that these orbitals can mix with the valence $2 \mathrm{~s} 2 \mathrm{p}$ orbitals of fluorine and oxygen, leading to large errors in the heat of formation of the corresponding binary compounds, such as $\mathrm{HfOF}_{2}$ or $\mathrm{HfF}_{2} \cdot{ }^{37-38}$ In general, since the same atom can behave differently in different situations, the question of which is the optimal number of electrons to be included in a computational investigation is still unsettled, making comparison between different systems a very tedious task.

In this work, we initially use an energy criterion for defining a conservative number of electrons that can be reasonably excluded from the correlation treatment of each atom, with the final aim of retaining the accuracy and the black-box applicability of the $\operatorname{CCSD}(\mathrm{T})$ method as well as the computational saving coming from the FC approximation. The validity of the suggested settings is tested for the calculation of heterolytic dissociation energies (HDEs) of the type $\mathrm{M} \rightarrow \mathrm{M}^{\mathrm{n}+}+\mathrm{nF}^{-}\left(\mathrm{M}^{\mathrm{n}+}=\mathrm{Li}^{+}, \mathrm{Na}^{+}, \mathrm{Al}^{3+}, \mathrm{Ca}^{2+}, \mathrm{Cu}^{+}, \mathrm{Ga}^{3+}, \mathrm{Y}^{3+}, \mathrm{Ag}^{+}, \mathrm{In}^{3+}\right.$, $\mathrm{Hf}^{4+}, \mathrm{Au}^{+}$) using canonical $\mathrm{CCSD}(\mathrm{T})$. Afterwards, the performance of the DLPNO-CCSD 
methodology in describing core-core, core-valence and valence-valence components of the correlation energy is investigated. As a result of this study, it was found that more conservative thresholds must be used for electron pairs containing core electrons in order to retain the accuracy that one is used to for valence calculations also for all-electron (AE) calculations. Finally, a comparison of DLPNO-CCSD and canonical CCSD results is given for the calculation of the same set of HDEs for both FC and AE calculations.

The paper is organized as follows. Computational details are given in Section 2. In Section 3.1, the new FC settings are defined and tested in the calculations of HDEs using canonical $\operatorname{CCSD}(\mathrm{T})$. In Section 3.2, the performance of the DLPNO-CCSD methodology for the calculation of core-core and core-valence correlation energies are discussed and the new methodology is described. Afterwards, the performance of canonical CCSD and DLPNOCCSD methods are compared for both AE and FC calculations.

\section{Computational details}

All calculations were performed with a development version (based on version 4.0) of the ORCA suite of programs. ${ }^{39}$

\subsection{Orbital energies}

Atomic orbital energies for the elements of the periodic table in their ground state (Section 3.1) were calculated at the Unrestricted Hartee-Fock (UHF) level of theory, using the zerothorder regular approximation $\left(\mathrm{ZORA}^{40}\right)$. For elements from Li to Kr, the ZORA-def2-tzvp basis set was used, which is a recontracted version of the original Ahlrichs' basis set. ${ }^{41}$ For the elements from Rb to I, a recontracted version of the TZVP basis set, namely "ZORA-TZVP", was used, as implemented in ORCA. For the elements heavier than Xe, SARC-ZORA-TZVP basis sets ${ }^{42,43}$ were used. 


\subsection{Heterolytic dissociation energies}

Geometry optimizations were carried out at the RI-MP2 level of theory, using correlation consistent core valence basis sets of triple- $\zeta$ quality, namely cc-pwCVTZ. ${ }^{37,}{ }^{44-47}$ For Y, Ag, In, Hf and Au, pseudopotential-based cc-pwCVTZ-PP basis sets have been used. ${ }^{48-49}$ The auxiliary basis set was generated using the AutoAux utility of ORCA, as described in Ref. ${ }^{50}$

For the sixth-period transition metals, all electrons outside the pseudopotential region were included in the correlation treatment. In all other cases, the "reduced FC settings" were applied, as detailed in Section 3.1. The optimized Cartesian coordinates of all the systems are given in the Supporting Information.

AE single point CCSD(T), CCSD and DLPNO-CCSD energies were calculated on RI-MP2 geometries. For reactions involving metals of the second, third and fourth period, the all electron cc-pwCV5Z ${ }^{44-48}$ basis set was used for all elements. For $\mathrm{Y}, \mathrm{Ag}, \mathrm{In}, \mathrm{Hf}$ and $\mathrm{Au}$, the second order Douglas-Kroll-Hess Hamiltonian was used in conjunction with the all electron cc-pwCVTZ-DK basis set. ${ }^{37,51-52}$ In the reactions involving these elements, the cc-pwCVTZ basis set was used for F.

AutoAux was used for the generation of the auxiliary basis set in DLPNO-CCSD calculations. The role of sub-valence correlation was estimated by using different FC settings in conjunction with the same basis set. In particular, the difference between the HDE obtained for a given FC setting and the one obtained at the $\mathrm{AE}$ level $(\triangle \mathrm{HDE})$ was calculated and discussed. Note that, for the studied reactions, the basis set superposition error $\left(\mathrm{BSSE}^{53}\right)$ can be significant, as shown in the Supporting Information (Table S1 and S2). The error is due to the fact that $\mathrm{F}^{-}$shows a very slow convergence of the energy with the basis set size, which can improve if diffuse functions are included in the calculation (Table S3 in the Supporting Information).${ }^{54}$ However, the focus of this work is the discussion of sub-valence correlation 
effects, and the central quantity of our discussion, $\triangle \mathrm{HDE}$, is only weakly affected by the BSSE correction, as shown in the Supporting Information (Table S1 and S2).

Unless otherwise specified, the DLPNO-CCSD calculations were carried out with TightPNO settings. ${ }^{22-23}$ With TightPNO thresholds $\left(\mathrm{T}_{\text {CutPairs }}=10^{-5}, \mathrm{~T}_{\text {CutPNO }}=10^{-7}, \mathrm{~T}_{\text {CutMKN }}=\right.$ $10^{-3}$ ), the fraction of the recovered correlation energy with respect to canonical coupled cluster is typically greater than $99.9 \%$. The Foster-Boys localization scheme ${ }^{55}$ was applied in all cases.

\section{Results and Discussion}

\subsection{New FC settings and sub-valence correlation effects}

Two fundamental aspects must be considered when FC calculations are performed: ${ }^{33}$ (i) the definition of the core space of each atom, (ii) the identification of $\mathrm{N}$ molecular orbitals (MOs), where $\mathrm{N}$ is the sum of core orbitals over all atoms, that correspond to the chosen definition of atomic core orbitals. Petersson and coworkers have successfully addressed the latter issue and proposed an overlap criterion for the identification of core MOs for any atomic core definition. ${ }^{33}$ A similar methodology for addressing the same problem has been implemented in ORCA and tested in our group ${ }^{36}$ and has been also applied in the present work. Initially, the $\mathrm{N}$ lowest energy MOs define the core region, whilst all MOs with higher orbital energy define the valence region. However, this ordering can lead to inversion of (physical) core and valence orbitals. Therefore, the routine outlined in Scheme 1 detects and repairs inversion of core and valence orbitals and detects mixing of core orbitals with core or valence orbitals. 
1 Compute sum of core orbitals C(A) for each atom type $\mathrm{A}$

2 Compute sum of all core orbitals C_all

3 Loop over all MOs i

4 Compute Mulliken population $M(A)$ per atom type for Mo $i$

5 Compute Mulliken population $M(A, 1)$ per atom type and

6 angular momentum 1 for MO i

7 Set atom type A(i) for which $M(A)$ is max

8 Set $M_{\text {max }}(i)$ as the maximum value of $M(\mathbb{A}, 1)$

9 Set actual number of core orbitals per atom type $c(A)$ to zero

10 Loop over all MOs i starting from lowest energy MO

11 is_core (i) = false

12 If $c(A)<C(A)$

13 is_core (i) = true

$14 \quad \mathrm{C}(\mathrm{A})=\mathrm{c}(\mathrm{A})+1$

$15 \quad$ If $\mathrm{M}_{\max }(i)<0.9$

16 Warning: "Significant orbital mixing for core Mo $1 . "$

17 For all Mos $i<=$ C_all check whether is_core(i) is true, for

18 all Mos $i>$ C_all check whether is_core(i) is false.

19 Invert the order of the MOs that are in the wrong region.

Scheme 1: Pseudocode for detecting and repairing inversion of core and valence orbitals and for detecting mixing of core orbitals with core or valence orbitals.

On the other hand, the definition of the core space can be difficult and the simplistic view that the core of each element can be assigned based on a casual inspection of the periodic 
table has already been found inadequate in a large number of situations, as already mentioned in the Introduction. In this section, a conservative definition for the core of each element to be used in standard $\operatorname{CCSD}(\mathrm{T})$ and $\operatorname{DLPNO}-\operatorname{CCSD}(\mathrm{T})$ applications is given, with the aim of conserving the black-box applicability of the CC method while retaining chemical accuracy (1 $\mathrm{kcal} / \mathrm{mol}$ ). We shall refer to this new core as "reduced core", whilst the remaining electrons of the same element are denoted hereafter as "extended valence"'.

In order to define the reduced core, we initially computed occupied atomic orbital energies for all elements from $\mathrm{Li}$ to $\mathrm{Rn}$ in their ground state, using UHF in conjunction with the ZORA Hamiltonian (see Section 2 for the computational details). For each element, orbitals with energy $<-200 \mathrm{eV}$ were included in the reduced core, while those with energy $>-80 \mathrm{eV}$ were included in the extended valence (See Figure 1). In those cases where the orbital energy was found within these two thresholds, we relied on some physically motivated assumptions, as we shall discuss in the following. In particular, a similar valence space was defined for elements of the same group. 


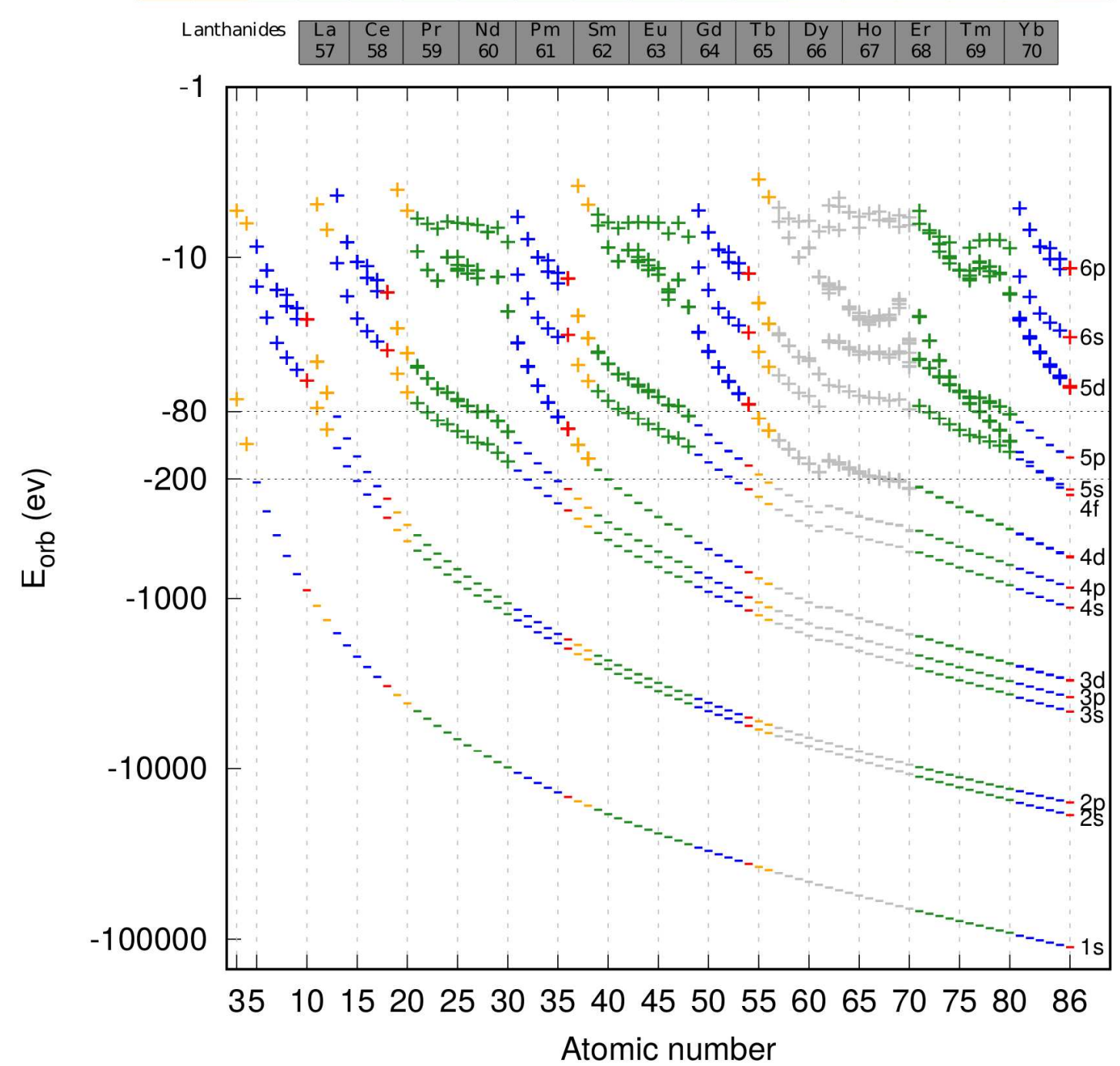

Figure 1: Orbital energies as a function of the atomic number. "+" denote extended-valence electrons, "-“" are reduced-core electrons. The color code is explained in the upper panel, , i.e. orange points denote s elements, green points transition metals of the $d$ block, blue points $p$ elements, red points noble gases and gray points the elements of the Lanthanide series. The atomic number of each element is also reported in the upper table for easy reference. See text for further details. 
In the lower panel of Figure 1, the energies of the (alpha) occupied orbitals for all the atoms included in the study are shown. The results of this study are summarized in Table 1, where the number of core electrons defined for each atom is reported.

Table 1: Core electrons suggested for coupled cluster calculations

\begin{tabular}{|l|l|l|}
\hline Elements & Reduced core electrons & Total number of electrons in the reduced core \\
\hline $\mathrm{H}-\mathrm{Be}$ & None & 0 \\
\hline $\mathrm{B}-\mathrm{Mg}$ & $1 \mathrm{~s}^{2}$ & 2 \\
\hline $\mathrm{Al}-\mathrm{Zn}$ & $1 \mathrm{~s}^{2} 2 \mathrm{~s}^{2} 2 \mathrm{p}^{6}$ & 10 \\
\hline Ga-Sr & $1 \mathrm{~s}^{2} 2 \mathrm{~s}^{2} 2 \mathrm{p}^{6} 3 \mathrm{~s}^{2} 3 \mathrm{p}^{6}$ & 18 \\
\hline $\mathrm{Y}-\mathrm{Cd}$ & $1 \mathrm{~s}^{2} 2 \mathrm{~s}^{2} 2 \mathrm{p}^{6} 3 \mathrm{~s}^{2} 3 \mathrm{p}^{6} 3 \mathrm{~d}^{10}$ & 28 \\
\hline In-Yb & $1 \mathrm{~s}^{2} 2 \mathrm{~s}^{2} 2 \mathrm{p}^{6} 3 \mathrm{~s}^{2} 3 \mathrm{p}^{6} 3 \mathrm{~d}^{10} 4 \mathrm{~s}^{2} 4 \mathrm{p}^{6}$ & 36 \\
\hline $\mathrm{Lu}-\mathrm{Hg}$ & $1 \mathrm{~s}^{2} 2 \mathrm{~s}^{2} 2 \mathrm{p}^{6} 3 \mathrm{~s}^{2} 3 \mathrm{p}^{6} 3 \mathrm{~d}^{10} 4 \mathrm{~s}^{2} 4 \mathrm{p}^{6} 4 \mathrm{~d}^{10}$ & 46 \\
\hline Tl-Rn & $1 \mathrm{~s}^{2} 2 \mathrm{~s}^{2} 2 \mathrm{p}^{6} 3 \mathrm{~s}^{2} 3 \mathrm{p}^{6} 3 \mathrm{~d}^{10} 4 \mathrm{~s}^{2} 4 \mathrm{p}^{6} 4 \mathrm{~d}^{10} 4 \mathrm{f}^{14} 5 \mathrm{~s}^{2} 5 \mathrm{p}^{6}$ & 68 \\
\hline
\end{tabular}

According to the energy criterion, for the s-block elements, electrons in the $n \mathrm{~s},(n-1) \mathrm{s}$ and ( $n$ 1)p orbitals are included in the extended valence, with $n$ being the highest principal quantum number among the occupied orbitals (i.e. $n=2$ for $\mathrm{Li}$ and $\mathrm{Be}, n=3$ for $\mathrm{Na}$ and $\mathrm{Mg}$ and so on...). This is consistent with the fact that the inclusion of the $(n-1) \mathrm{s}$ and $(n-1) \mathrm{p}$ (outer-core) shells in the correlation space of alkaline and alkaline-earth metals has already been found to be important in a wide range of situations. ${ }^{25,27,36}$ Note that the proposed extended valence definition requires the use of appropriate core-valence basis functions for the correlation of the $(n-1)$ s and $(n-1)$ electrons. ${ }^{46}$

For the p-block elements, $n \mathrm{~s}, n \mathrm{p}$ and $(n-1) \mathrm{d}$ electrons (if $n \geq 4)$ are included in the extended valence. For B-Ne and $\mathrm{Al}-\mathrm{Cr}$ this definition corresponds to the standard definition of valence, whilst for Ga-Kr, In-Xe and Tl-Rn the $(n-1) \mathrm{d}$ orbitals are also included. Note that the importance of correlating the $3 \mathrm{~d}$ electrons in the calculation of atomization energies of $\mathrm{Ga}$ oxides and fluorides has already been documented. ${ }^{29,}{ }^{56}$ It is worth mentioning that the correlation of the $(n-1)$ d electrons requires the use of appropriate basis functions, which are 
usually not included in standard "valence" basis sets. ${ }^{56}$ For example, widely availabe Ga basis sets in the literature as cc-pVnZ, cc-pVnZ-DK, cc-pVnZ-PP, def2-nZVP, 6-311G(d,p), etc. have only been designed for valence $n s p$ correlation. To correlate the outer-core $(n-1) \operatorname{spd}$ electrons in Ga, specifically designed basis sets, as cc-pwCVnZ-DK, have to be used.

For the d-block transition metals, $n \mathrm{~s},(n-1) \mathrm{s},(n-1) \mathrm{p}$ and $(n-1) \mathrm{d}$ orbitals are included in the extended valence ( $n=4,5$ and 6 for the fourth, fifth and sixth period, respectively). For the transition metals of the sixth period, we decided to include also the $4 \mathrm{f}$ orbitals in the extended valence, because they are energetically very close to the $5 \mathrm{~s}$ and $5 \mathrm{p}$ orbitals. As already mentioned in the introduction, the relativistic Stuttgart-Dresden effective core potential for sixth-period transition metals includes the $4 \mathrm{f}$ electrons. Therefore, the only practical way of including dynamic correlation from the $4 \mathrm{f}$ orbitals is the use of a relativistic Hamiltonian. Note that a series of correlation consistent core-valence basis sets directly optimized for including electron correlation from the $4 \mathrm{f}$ orbitals is not yet available in literature (the ccpwCVTZ(+4f)-DK basis set is available for some of the transition metals of the sixth period $^{37}$ ). Hence, rigorous extrapolation to the basis set limit with $4 \mathrm{f}$ electrons correlated is not possible. This can limit the accuracy of coupled cluster predictions, especially if one targets tighter than so-called chemical accuracy. However, the development of new correlation consistent basis sets is beyond the scope of the present work.

Regarding the Lanthanides series, we decide to apply the more conservative energy criterion $(-200 \mathrm{eV})$, and hence the extended valence includes the $4 \mathrm{~d}, 4 \mathrm{f}, 5 \mathrm{~s}, 5 \mathrm{p}$ and the $5 \mathrm{~d}$ shells.

These FC settings have been tested in a series of heterolytic dissociation energies (HDEs) for reactions of the type $\mathrm{MF}_{\mathrm{n}} \rightarrow \mathrm{M}^{\mathrm{n}+}+\mathrm{nF}^{-}\left(\mathrm{M}^{\mathrm{n}+}=\mathrm{Li}^{+}, \mathrm{Na}^{+}, \mathrm{Al}^{3+}, \mathrm{Ca}^{2+}, \mathrm{Ga}^{3+}, \mathrm{Cu}^{+}, \mathrm{Y}^{3+}, \mathrm{Ag}^{+}\right.$, $\left.\mathrm{Hf}^{4+}, \mathrm{Au}^{+}\right)$. For this type of reactions, sub-valence correlation effects are expected to play an important role due to the high ionicity of the bonds and the small number of electrons that 
some of the metals have in the valence. The HDE calculated at the $\operatorname{CCSD}(\mathrm{T})$ level with different FC settings for the metal are reported in Table 2. In the same Table, the difference between the HDE obtained for a given FC setting and the one obtained at the AE level $(\triangle \mathrm{HDE})$ is reported. Note that the $1 \mathrm{~s}^{2}$ electrons of $\mathrm{F}$ were excluded from the correlation treatment in all FC calculations, whilst all electrons of $\mathrm{F}$ were included in the correlation treatment in $\mathrm{AE}$ calculations.

Table 2: Calculated heterolytic dissociation energies (HDE in $\mathrm{kcal} / \mathrm{mol}$ ) for $\mathrm{MF}_{\mathrm{n}}$ compounds $\left(\mathrm{MF}_{\mathrm{n}} \rightarrow \mathrm{M}^{\mathrm{n}+}+\mathrm{nF}^{-}\right)$at the canonical CCSD(T) level of theory with different FC settings for the metal. See text for details.

\begin{tabular}{|c|c|c|c|c|}
\hline $\mathrm{MF}_{\mathrm{n}}$ & Entry & Core-electrons of $\mathrm{M}$ in the correlation treatment & HDE & $\triangle \mathrm{HDE}$ \\
\hline \multicolumn{5}{|c|}{ s-block metal } \\
\hline \multirow{2}{*}{$\mathrm{LiF}$} & 1 & None & 190.1 & 0.0 \\
\hline & 2 & $1 \mathrm{~s}^{2}$ & 187.9 & -2.1 \\
\hline \multirow{3}{*}{$\mathrm{NaF}$} & 3 & None & 159.4 & 0.0 \\
\hline & 4 & $1 \mathrm{~s}^{2}$ & 159.4 & 0.0 \\
\hline & 5 & $1 s^{2} 2 s^{2} 2 p^{6}$ & 154.7 & -4.8 \\
\hline \multirow[b]{3}{*}{$\mathrm{CaF}_{2}$} & 6 & None & 537.3 & 0.0 \\
\hline & 7 & $1 s^{2} 2 s^{2} 2 p^{6}$ & 536.6 & -0.7 \\
\hline & 8 & $1 s^{2} 2 s^{2} 2 p^{6} 3 s^{2} 3 p^{6}$ & 506.6 & -30.7 \\
\hline \multicolumn{5}{|c|}{ p-block metal } \\
\hline \multirow{2}{*}{$\mathrm{AlF}_{3}$} & 9 & None & 1436.2 & 0.0 \\
\hline & 10 & $1 s^{2} 2 s^{2} 2 p^{6}$ & 1424.5 & -11.6 \\
\hline \multirow{2}{*}{$\mathrm{GaF}_{3}$} & 11 & None & 1423.2 & 0.0 \\
\hline & 12 & $1 s^{2} 2 s^{2} 2 p^{6} 3 d^{10} 3 s^{2} 3 p^{6}$ & 1422.5 & -0.7 \\
\hline \multirow{2}{*}{$\mathrm{InF}_{3}$} & 13 & None & 1338.2 & 0.0 \\
\hline & 14 & $1 s^{2} 2 s^{2} 2 p^{6} 3 d^{10} 3 s^{2} 3 p^{6} 3 d^{10} 4 s^{2} 4 p^{6}$ & 1335.0 & -3.2 \\
\hline \multicolumn{5}{|c|}{ d-block metal } \\
\hline \multirow{2}{*}{$\mathrm{CuF}$} & 15 & None & 196.6 & 0.0 \\
\hline & 16 & $1 s^{2} 2 s^{2} 2 p^{6}$ & 196.5 & -0.1 \\
\hline \multirow{2}{*}{$\mathrm{YF}_{3}$} & 17 & None & 1200.8 & 0.0 \\
\hline & 18 & $1 s^{2} 2 s^{2} 2 p^{6} 3 s^{2} 3 p^{6} 3 d^{10}$ & 1199.5 & -0.1 \\
\hline \multirow{2}{*}{$\mathrm{AgF}$} & 19 & None & 195.9 & 0.0 \\
\hline & 20 & $1 s^{2} 2 s^{2} 2 p^{6} 3 s^{2} 3 p^{6} 3 d^{10}$ & 195.6 & -0.3 \\
\hline \multirow{3}{*}{$\mathrm{HfF}_{4}$} & 21 & None & 2193.9 & 0.0 \\
\hline & 22 & $1 s^{2} 2 s^{2} 2 p^{6} 3 s^{2} 3 p^{6} 3 d^{10} 4 s^{2} 4 p^{2} 4 d^{10}$ & 2192.9 & -1.0 \\
\hline & 23 & $1 s^{2} 2 s^{2} 2 p^{6} 3 s^{2} 3 p^{6} 3 d^{10} 4 s^{2} 4 p^{2} 4 d^{10} 4 f^{14}$ & 2156.1 & -37.7 \\
\hline $\mathrm{AuF}$ & 24 & None & 223.6 & 0.0 \\
\hline
\end{tabular}




\begin{tabular}{|l|l|l|l|l|}
\hline & $\mathbf{2 5}$ & $1 \mathrm{~s}^{2} 2 \mathrm{~s}^{2} 2 \mathrm{p}^{6} 3 \mathrm{~s}^{2} 3 \mathrm{p}^{6} 3 \mathrm{~d}^{10} 4 \mathrm{~s}^{2} 4 \mathrm{p}^{2} 4 \mathrm{~d}^{10}$ & 223.0 & -0.6 \\
\cline { 2 - 5 } & $\mathbf{2 6}$ & $1 \mathrm{~s}^{2} 2 \mathrm{~s}^{2} 2 \mathrm{p}^{6} 3 \mathrm{~s}^{2} 3 \mathrm{p}^{6} 3 \mathrm{~d}^{10} 4 \mathrm{~s}^{2} 4 \mathrm{p}^{2} 4 \mathrm{~d}^{10} 4 \mathrm{f}^{14}$ & 223.3 & -0.3 \\
\hline
\end{tabular}

For reactions involving s-block metals, we computed the $\mathrm{HDE}$ of $\mathrm{LiF}, \mathrm{NaF}$ and $\mathrm{CaF}_{2}$. In all cases, FC calculations using the reduced core definition (reduced FC settings) give $\Delta \mathrm{HDE}$ lower than $1 \mathrm{kcal} / \mathrm{mol}$ (entry $\mathbf{1 , 4}$ and 7). It is worth mentioning that the correlation consistent core-valence basis sets used here are optimized for correlating the valence and the outer-core electrons from the $(n-1) \mathrm{s}$ and $(n-1) \mathrm{p}$ orbitals. Therefore, at least part of the residual $\Delta \mathrm{HDE}$ observed for entry 7 may be due to the use of this basis set in AE calculations. On the other hand, excluding the sub-valence $(n-1) \mathrm{s}$ and $(n-1) \mathrm{p}$ orbitals from the correlation treatment leads to much larger deviations in all cases (entry $\mathbf{2 , 5}$ and $\mathbf{8}$ ). In particular, a strong orbital mixing, as defined by Mulliken population analysis, was found between the $3 p$ orbitals of Ca with the 2s orbitals of fluorine in $\mathrm{CaF}_{2}$, consistently with recently published results. ${ }^{36}$ Accordingly, the exclusion of the $3 p(\mathrm{Ca})$ orbitals from the correlation treatment led to a $\triangle$ HDE of -30.7 $\mathrm{kcal} / \mathrm{mol}$ (entry 8). For the other elements of the s-block, no significant orbital mixing was detected between the $(n-1) \mathrm{p}$ shell of $\mathrm{M}$ and the $2 \mathrm{~s}$ orbitals of F. Nevertheless, significant subvalence correlation effects are present for both $\mathrm{LiF}$ and $\mathrm{NaF}$ (entry $\mathbf{2}$ and $\mathbf{5}$ ), probably arising from neglecting the attractive dispersion interaction between the electrons in the fluorine and the ones in the core of the metal. Note that similar results were found for the homolytic dissociation of the same species (Table S4 in the Supporting Information), even if the FC error due to the inclusion of the $(n-1) \mathrm{s}$ and $(n-1) \mathrm{p}$ orbitals in the core is significantly smaller in this case. It is also worth mentioning that similar sub-valence correlation effects were found for the heterolytic dissociation of the analogous chlorinated species $\left(\mathrm{LiCl}, \mathrm{NaCl}\right.$ and $\left.\mathrm{CaCl}_{2}\right)$, as shown the Supporting Information (Table S5). All these results are consistent with the ones reported recently, ${ }^{36}$ and demonstrate that the reduced FC settings can be safely applied for alkaline and alkaline earth metals compounds in these reactions. 
For p-block metal compounds, a significant sub-valence correlation effect was found in the case of $\mathrm{AlF}_{3}$, giving a $\triangle \mathrm{HDE}$ of $-11.6 \mathrm{kcal} / \mathrm{mol}$ (entry 10), despite no significant orbital mixing was found. Conversely, in the case of $\mathrm{GaF}_{3}$ (entry 12), sub-valence correlation does not seem to play an important role. For $\mathrm{InF}_{3}$, the effect of sub-valence correlation is again substantial, with $\triangle \mathrm{HDE}$ of $-3.2 \mathrm{kcal} / \mathrm{mol}$ (entry 14). Remarkably enough, these differences correlate with the energies of the $(n-1)$ p orbitals of the ground state of the free metal shown in Figure $1(-80 \mathrm{eV}, 123 \mathrm{eV}$ and $97 \mathrm{eV}$ for $\mathrm{Al}, \mathrm{Ga}$ and $\mathrm{In}$, respectively) which are the highestenergy orbitals excluded from the correlation treatment. These results would suggest to include the $(n-1) \mathrm{s}$ and $(n-1) \mathrm{p}$ orbitals in the reduced core also for p-block elements. However, it is worth stressing here that, by increasing the atomic number for elements of the same period, the energy of the $(n-1)$ p shell decreases significantly. For instance, going from Al to $\mathrm{Si}$, the energy of the $2 \mathrm{p}$ orbitals of the ground state of the free atoms decreases from $-80 \mathrm{eV}$ to $-116 \mathrm{eV}$, and keeps decreasing afterwards, as shown in Figure 1. Therefore, for p-block elements, the $(n-1) \mathrm{s}$ and $(n-1) \mathrm{p}$ orbitals are included in the reduced core, but we recommend to check the results of FC calculations when early p-block elements of the third period are present.

For d-block metal compounds, the reduced FC settings always lead to errors $<1 \mathrm{kcal} / \mathrm{mol}$ in all cases (entries 16, 18, 20, 22 and 24). Of particular interest is the effect of the $4 \mathrm{f}$ electrons for transition metals of the sixth period. In the case of $\mathrm{HfF}_{4}$, the $4 \mathrm{f}$ orbitals mix with the ones in the fluorine atoms, leading to a very large $\triangle \mathrm{HDE}$ if these are excluded from the correlation treatment (entry 23). Interestingly, in the case of AuF, these orbitals do not play any particular role (entry $\mathbf{2 4 ,} 25$ and $\mathbf{2 6}$ show practically the same results). The difference between Au and Hf can be explained in terms of the energy of the $4 \mathrm{f}$ orbitals in the two situations. In the Hf case, the $4 \mathrm{f}$ orbitals are very high in energy (about $-31 \mathrm{eV}$ ), whilst for Au the energy of these orbitals is about $-100 \mathrm{eV}$. However, since the $4 \mathrm{f}$ orbitals are relatively high in energy for all 
the sixth-period transition metals, we suggest to include these orbitals in the correlation treatment for black-box $\operatorname{CCSD}(\mathrm{T})$ applications.

\subsection{Core correlation in the DLPNO-CCSD framework}

In the previous section, we used an energy criterion for determining a conservative number of electrons that can be excluded from the correlation treatment for standard chemical applications. However, due to the infinite number of possible applications, there could be cases for which AE calculations cannot be avoided. In these cases, the DLPNO-CCSD method permits the inclusion of core electrons in the correlation treatment without a dramatic increase in the computational cost. ${ }^{36}$

In this Section, the performance of the DLPNO-CCSD method in describing the core-core, core-valence and valence-valence components of the correlation energy is carefully analyzed. For "core", we refer to the "reduced core" shown in Table 1 and defined in section 3.1. Analogously, for "valence", we refer to the "extended valence" defined in the same section. Before starting our discussion, we shall recall the most important features of the DLPNOCCSD method for the sake of clarity.

In the DLPNO-CCSD method, the correlation energy is obtained as a sum over electron pair contributions $\varepsilon_{i j}$, with $\mathrm{i}$ and $\mathrm{j}$ the localized orbitals of the reference determinant, plus a perturbative triple correction. ${ }^{21}$ The $\varepsilon_{i j}$ terms are divided into "weak-pairs", treated at the local MP2 (LMP2) level and with expected negligible contribution, and "strong-pairs", treated by CCSD in the PNOs framework. In the PNO coupled cluster equations, the virtual space is spanned by a highly compact set of PNOs that are specific for each electron pair and are obtained upon diagonalization of LMP2 pair density matrices. Only PNOs with occupation number larger than $\mathrm{T}_{\mathrm{CutPNO}}$ are kept. Despite the dependence of the correlation energy on the $\mathrm{T}_{\text {CutPNO }}$ parameter has already been carefully analyzed using the $\mathrm{FC}$ 
approximation, ${ }^{22-23}$ the way this parameter influences core-core and core-valence contributions was not discussed before.

As a prototype case study, we initially focus our attention on the $\mathrm{Ca}^{2+}$ ion. In particular, we investigate the dependence of the DLPNO-CCSD/cc-pwCV5Z core-core, core-valence and valence-valence components of the correlation energy on the $\mathrm{T}_{\text {CutPNO }}$ threshold. As reference values, we used DLPNO-CCSD energies corresponding to $\mathrm{T}_{\mathrm{CutPNO}}=0$, i.e. all PNOs are included in the calculation.

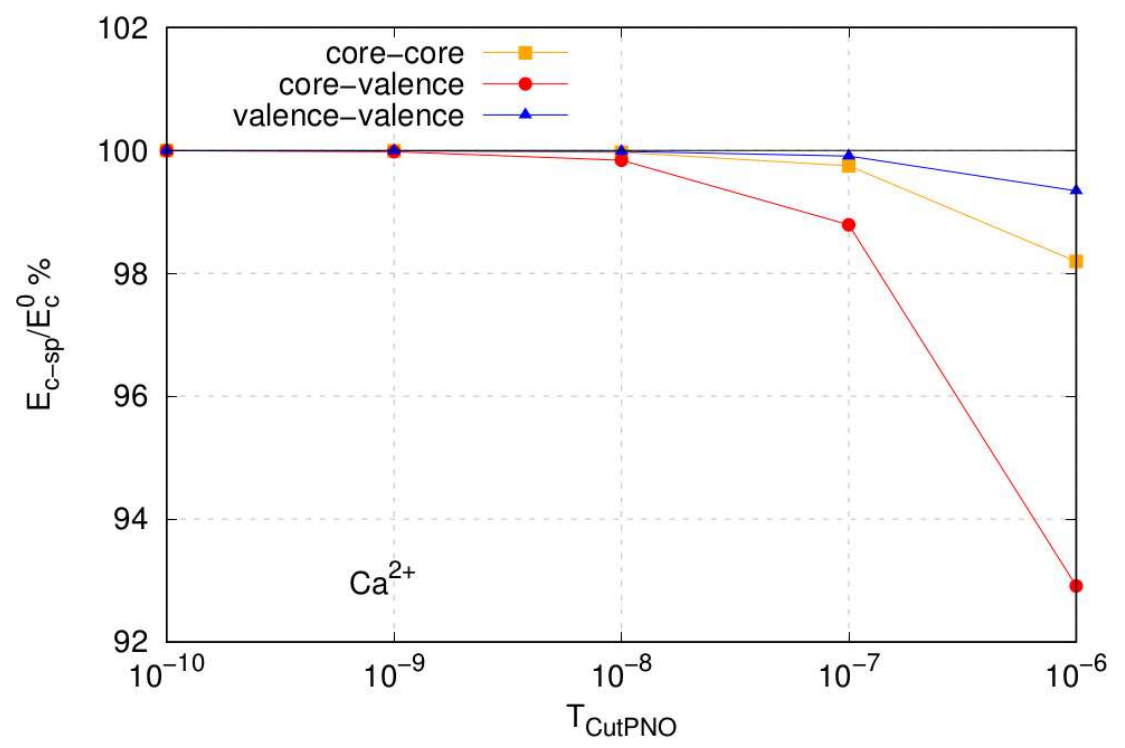

Figure 2: Percentage of core-core, core-valence and valence-valence correlation energy contributions recovered by the strong-pairs for different $\mathrm{T}_{\mathrm{CutPNO}}$ thresholds for $\mathrm{Ca}^{2+}$. As reference values, DLPNO-CCSD results using $\mathrm{T}_{\mathrm{CutPNO}}=0$ for all electron pairs were used.

The percentage of different components of the correlation energy recovered by the strongpairs with respect to the reference value is shown in Figure 2 as a function of $\mathrm{T}_{\text {CutPNO}}$. More than $99.9 \%$ of all the components of the correlation energy is recovered by the strong-pairs when $\mathrm{T}_{\mathrm{CutPNO}}=10^{-8}$ is used. However, the percentage becomes much smaller for the core-core and core-valence components for $\mathrm{T}_{\mathrm{CutPNO}}=10^{-7}$, and decreases dramatically for $\mathrm{T}_{\mathrm{CutPNO}}=10^{-6}$ (note that $10^{-7}$ and $10^{-6}$ are the default $\mathrm{T}_{\mathrm{CutPNO}}$ values used for TightPNO and LoosePNO 
settings, respectively ${ }^{23}$ ). This behavior is due to the large energy separation between the electrons in the reduced core and the virtual orbitals, which causes the corresponding LMP2 amplitudes to vanish. Since these amplitudes are used to build the pair density matrix from which the PNOs are generated, electron pairs involving core electrons typically feature PNOs with lower occupation numbers, which are screened out by the $\mathrm{T}_{\text {CutPNO }}$ threshold.

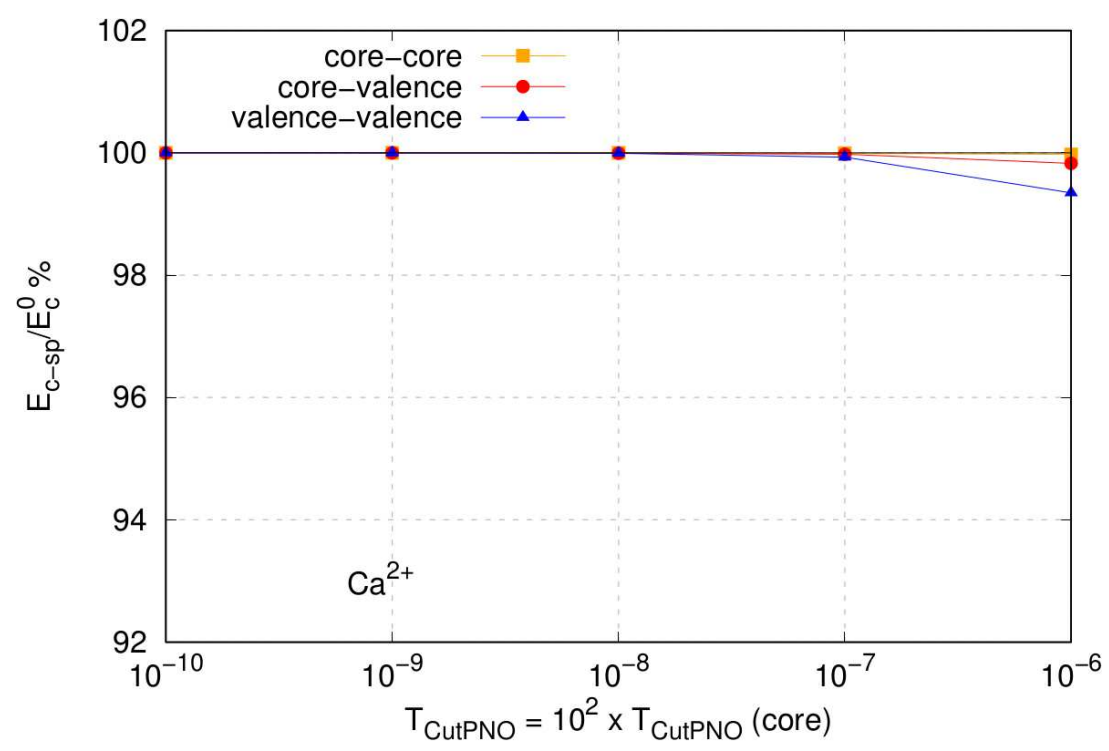

Figure 3 Percentage of core-core, core-valence and valence-valence correlation energy recovered by the strong-pairs for different $\mathrm{T}_{\mathrm{CutPNO}}$ thresholds for $\mathrm{Ca}^{2+}$. For the reduced-core

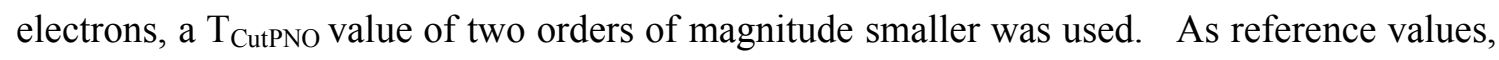
DLPNO-CCSD results using $\mathrm{T}_{\mathrm{CutPNO}}=0$ for all electron pairs were used.

It is worth mentioning here that most of the truncation error observed is usually well recovered by second order perturbation theory, and so the overall DLPNO-CCSD energy can be close to the one obtained by canonical CCSD also in AE calculations. However, as we shall show in the following, an unbalanced description of the different components of the correlation energy might lead to unpredictable errors. A straightforward solution to this issue is the use of more conservative $\mathrm{T}_{\mathrm{CutPNO}}$ thresholds for electron pairs containing at least one core electron. On the technical side, this requires to localize separately core and valence 
orbitals and to use different thresholds for the two sets. From the results shown in Figure 2,

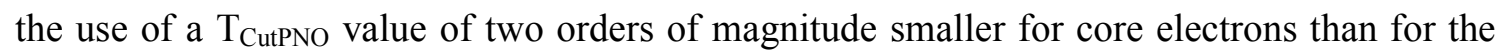
valence electrons appear to be a conservative choice. The results obtained in the $\mathrm{Ca}^{2+}$ case upon tightening of the thresholds for the core electrons are shown in Figure 3. With these settings, more than 99.9 percent of the core-core and core-valence correlation energy components is now recovered in all cases. The procedure just described is now the default in AE DLPNO-CCSD calculations for ORCA 4.0. For instance, if $\mathrm{T}_{\mathrm{CutPNO}}=10^{-7}$ is specified for the valence electrons, a $\mathrm{T}_{\mathrm{CutPNO}}$ value of $10^{-9}$ is used for the core-core and core-valence pairs (these values correspond to the current ORCA default for TightPNO settings).

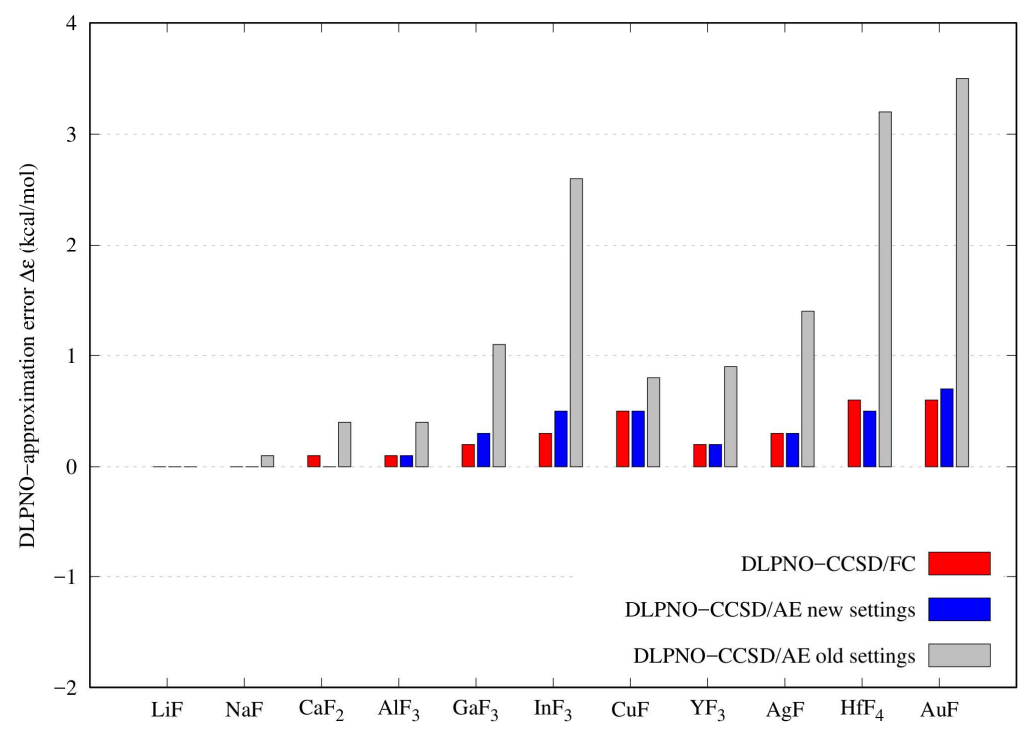

Figure 4: Error associated with the DLPNO approximation for the heterolityc dissociation energy of a series of fluorinated compounds for FC (DLPNO-CCSD/FC) and AE (DLPNOCCSD/AE) calculations. Canonical CCSD results are used as reference. "old settings" refers to $\mathrm{T}_{\mathrm{CutPNO}}=10^{-7}$ for all electron pairs, "new settings" refers to $\mathrm{T}_{\mathrm{CutPNO}}=10^{-7}$ for valence electron pairs and $\mathrm{T}_{\mathrm{CutPNO}}=10^{-9}$ for core-core and core-valence pairs. See Section 2.1 for the computational details.

We end this section with a comparison of DLPNO-CCSD and CCSD for the HDE defined in 
Section 3.1. The error in the HDE associated with the DLPNO approximation $(\Delta \varepsilon$, defined as the difference between DLPNO-CCSD and canonical CCSD reaction energies using the same number of electrons in the correlation treatment) is reported in Figure 4 for all reactions (the corresponding data are reported in the Supporting Information, Table S6). Red bars refer to $\Delta \varepsilon$ values calculated using reduced FC settings, blue bars the ones obtained for AE calculations with the new $\mathrm{T}_{\text {CutPNO }}$ settings (i.e. tightening the $\mathrm{T}_{\text {CutPNO }}$ thresholds for core-core and corevalence electron pairs) and gray bars denote the ones obtained for AE calculations with the old $\mathrm{T}_{\text {CutPNO }}$ settings (i.e. the same $\mathrm{T}_{\text {CutPNO }}$ is used for all pairs). The average $\Delta \varepsilon$ obtained for $\mathrm{FC}$ and $\mathrm{AE}$ calculations with the new $\mathrm{T}_{\text {CutPNO }}$ settings is less than $0.3 \mathrm{kcal} / \mathrm{mol}$, with a maximum deviation of about $0.5 \mathrm{kcal} / \mathrm{mol}$ in the AuF case. Conversely, AE calculations with the old settings show much larger $\Delta \varepsilon$ in all cases, with a maximum of $3.5 \mathrm{kcal} / \mathrm{mol}$ for AuF. For this critical case, the AE DLPNO-CCSD correlation energy (strong pairs + weak pairs) of AuF with the old DLPNO settings is -1.6733 a.u., which corresponds to $97.7 \%$ of the canonical CCSD correlation energy. With the new DLPNO settings, the correlation energy is 1.7083 a. u., which corresponds to $99.8 \%$ of the canonical result. FC DLPNO-CCSD calculations recover more than $99.9 \%$ of the corresponding FC CCSD correlation energy in this case.

Note that, in AE DLPNO-CCSD calculations, tightening of the PNO threshold for the reduced core electrons causes a moderate increase in the computational cost from $3280 \mathrm{~s}$ to $4493 \mathrm{~s}(+32 \%$, using 8 cores of a single node with two 8-core Intel Xeon E5-2670 CPUs and 256 GB of main memory). The same calculation carried out with canonical CCSD requires $41108 \mathrm{~s}$.

It is worth underscoring that, with the new settings, AE DLPNO-CCSD calculations use, by default, the definition of the reduced core to determine the electron pairs for which more conservative thresholds have to be used. In our opinion, this is a reasonable choice as the 
validity of both approximations (the FC approximation and the PNO truncation) ultimately depends on the orbital energy. However, as discussed in Section 3.1, for the standard chemical applications, electrons in the reduced core can be safely excluded from the correlation treatment, thus permitting one to avoid the increase in the computational cost that results from correlating the electrons in the reduced core.

\section{Conclusions}

For all the elements of the periodic table up to $\mathrm{Rn}$, we defined the "reduced core" as the number of electrons that can be excluded from the correlation treatment in practical CCSD(T) and DLPNO-CCSD(T) calculations while retaining chemical accuracy. To do that, we computed atomic orbital energies for the elements of the periodic table from Li to $\mathrm{Rn}$, and we used an energy criterion for determining which orbitals to include in the core. Afterwards, we tested the validity of these settings in the calculation of the heterolytic dissociation energy (HDE) of a series of fluorinated metal compounds, for which sub-valence correlation effects play an important role. It has been found that frozen-core (FC) calculations can be performed in a black-box fashion without significant loss of accuracy with respect to AE calculations. The suggested FC settings are reported in Table 1.

Having defined the optimal number of electrons to be included in the correlation treatment, we investigated the performance of the DLPNO-CCSD methodology in describing the different components of the correlation energy, namely the core-core, core-valence and valence-valence components. It was found that a more conservative $\mathrm{T}_{\mathrm{CutPNO}}$ threshold must be used for electron pairs containing at least one core electron in order to retain high accuracy in all-electron DLPNO-CCSD calculations. The new methodology was described and tested in the calculation of HDEs and it was found that DLPNO-CCSD reproduces canonical CCSD results for both all-electron and FC calculations. 


\section{Acknowledgment}

Giovanni Bistoni, Alexander A. Auer and Frank Neese gratefully acknowledge the Priority

Program "Control of London Dispersion Interactions in Molecular Chemistry"' (SPP 1807) of

the DFG for financial support. Yury Minenkov and Luigi Cavallo gratefully acknowledge the financial support from King Abdullah University of Science and Technology (KAUST).

\section{Supporting Information}

Further Tables and optimized geometries are included in the Supporting Information.

\section{Bibliography}

1. Bartlett, R. J.; Musial, M., Coupled-cluster Theory in Quantum Chemistry. Rev. Mod. Phys. 2007, 79, 291-352.

2. Feller, D.; Dixon, D. A., Extended Benchmark Studies of Coupled Cluster Theory through Triple Excitations. J. Chem. Phys. 2001, 115, 3484-3496.

3. Fang, Z.; Vasiliu, M.; Peterson, K. A.; Dixon, D. A., Prediction of Bond Dissociation Energies/Heats of Formation for Diatomic Transition Metal Compounds: CCSD(T) Works. J. Chem. Theory. Comput. 2017, 13, 1057-1066.

4. $\quad$ Cheng, L.; Gauss, J.; Ruscic, B.; Armentrout, P. B.; Stanton, J. F., Bond Dissociation Energies for Diatomic Molecules Containing 3d Transition Metals: Benchmark Scalar-Relativistic CoupledCluster Calculations for 20 Molecules. J. Chem. Theory. Comput. 2017, 13, 1044-1056.

5. $\quad$ Schutz, M.; Yang, J.; Chan, G. K.; Manby, F. R.; Werner, H. J., The Orbital-Specific Virtual Local Triples Correction: OSV-L(T). J. Chem. Phys. 2013, 138, 054109.

6. Schutz, M.; Werner, H. J., Low-Order Scaling Local Electron Correlation Methods. IV Linear Scaling Local Coupled-Cluster (LCCSD). J. Chem. Phys. 2001, 114, 661-681.

7. Schutz, M.; Werner, H. J., Local Perturbative Triples Correction (T) with Linear Cost Scaling. Chem. Phys. Lett. 2000, 318, 370-378.

8. Schutz, M.; Hetzer, G.; Werner, H. J., Low-Order Scaling Local Electron Correlation Methods. I. Linear Scaling Local MP2. J. Chem. Phys. 1999, 111, 5691-5705.

9. Hampel, C.; Werner, H.-J., Local Treatment of Electron Correlation in Coupled Cluster Theory. J. Chem. Phys. 1996, 104, 6286-6297.

10. Saebø, S.; Pulay, P., Local Configuration-Interaction - an Efficient Approach for Larger Molecules. Chem. Phys. Lett. 1985, 113, 13-18.

11. Saebø, S.; Pulay, P., 4th-Order Moller-Plessett Perturbation-Theory in the Local Correlation Treatment .1. Method. J. Chem. Phys. 1987, 86, 914-922.

12. Saebø, S.; Pulay, P., The Local Correlation Treatment .2. Implementation and Tests. J. Chem. Phys. 1988, 88, 1884-1890.

13. Saebø, S.; Pulay, P., Local Treatment of Electron Correlation. Annu. Rev. Phys. Chem. 1993, 44, 213-236. 
14. Neese, F.; Wennmohs, F.; Hansen, A., Efficient and Accurate Local Approximations to Coupled-Electron Pair Approaches: An Attempt to Revive the Pair Natural Orbital Method. J. Chem. Phys. 2009, 130, 114108.

15. Neese, F.; Hansen, A.; Wennmohs, F.; Grimme, S., Accurate Theoretical Chemistry with Coupled Pair Models. Acc. Chem. Res. 2009, 42, 641-648.

16. Neese, F.; Hansen, A.; Liakos, D. G., Efficient and Accurate Approximations to the Local Coupled Cluster Singles Doubles Method Using a Truncated Pair Natural Orbital Basis. J. Chem. Phys. 2009, 131, 064103.

17. Hansen, A.; Liakos, D. G.; Neese, F., Efficient and Accurate Local Single Reference Correlation Methods for High-Spin Open-Shell Molecules Using Pair Natural Orbitals. J. Chem. Phys. 2011, 135, 214102-20.

18. Liakos, D. G.; Hansen, A.; Neese, F., Weak Molecular Interactions Studied with Parallel Implementations of the Local Pair Natural Orbital Coupled Pair and Coupled Cluster Methods. J. Chem. Theory. Comput. 2011, 7, 76-87.

19. Huntington, L. M.; Hansen, A.; Neese, F.; Nooijen, M., Accurate Thermochemistry from a Parameterized Coupled-Cluster Singles and Doubles Model and a Local Pair Natural Orbital Based Implementation for Applications to Larger Systems. J. Chem. Phys. 2012, 136, 064101.

20. Riplinger, C.; Neese, F., An Efficient and near Linear Scaling Pair Natural Orbital Based Local Coupled Cluster Method. J. Chem. Phys. 2013, 138, 034106.

21. Riplinger, C.; Sandhoefer, B.; Hansen, A.; Neese, F., Natural Triple Excitations in Local Coupled Cluster Calculations with Pair Natural Orbitals. J. Chem. Phys. 2013, 139, 134101.

22. Riplinger, C.; Pinski, P.; Becker, U.; Valeev, E. F.; Neese, F., Sparse Maps-a Systematic Infrastructure for Reduced-Scaling Electronic Structure Methods. Ii. Linear Scaling Domain Based Pair Natural Orbital Coupled Cluster Theory. J. Chem. Phys. 2016, 144, 024109.

23. Liakos, D. G.; Sparta, M.; Kesharwani, M. K.; Martin, J. M. L.; Neese, F., Exploring the Accuracy Limits of Local Pair Natural Orbital Coupled-Cluster Theory. J. Chem. Theory. Comput. 2015, 11, 1525-1539.

24. Bistoni, G.; Auer, A. A.; Neese, F., Understanding the Role of Dispersion in Frustrated Lewis Pairs and Classical Lewis Adducts: A Domain-Based Local Pair Natural Orbital Coupled Cluster Study. Chemistry - A European Journal 2017, 23, 865-873.

25. Iron, M. A.; Oren, M.; Martin, J. M. L., Alkali and Alkaline Earth Metal Compounds: CoreValence Basis Sets and Importance of Subvalence Correlation. Mol Phys 2003, 101, 1345-1361.

26. Feller, D.; Peterson, K. A.; Dixon, D. A., Further Benchmarks of a Composite, Convergent, Statistically Calibrated Coupled-Cluster-Based Approach for Thermochemical and Spectroscopic Studies. Mol Phys 2012, 110, 2381-2399.

27. Sullivan, M. B.; Iron, M. A.; Redfern, P. C.; Martin, J. M. L.; Curtiss, L. A.; Radom, L., Heats of Formation of Alkali Metal and Alkaline Earth Metal Oxides and Hydroxides: Surprisingly Demanding Targets for High-Level Ab Initio Procedures. J. Phys. Chem. A 2003, 107, 5617-5630.

28. Schulz, A.; Smith, B. J.; Radom, L., Heats of Formation of Alkali and Alkaline Earth Oxides and Hydroxides: Some Dramatic Failures of the G2 Method. J. Phys. Chem. A 1999, 103, 7522-7527.

29. Bauschlicher, C. W., Jr.; Melius, C. F.; Allendorf, M. D., Gallium Compounds, a Possible Problem for the G2 Approaches. J. Chem. Phys. 1999, 110, 1879-1881.

30. Duke, B. J.; Radom, L., Gaussian-2 (G2) Theory for Third-Row Elements: A Systematic Study of the Effect of the 3d Orbitals. J. Chem. Phys. 1998, 109, 3352-3359.

31. Yamaguchi, Y.; Schaefer III, H. F., The GeOH-HGeO System: Are the 3d Electrons Core or Valence? J. Chem. Phys. 1996, 104, 9841-9847.

32. Richards, C. A. Jr .; Yamaguchi, Y.; Kim, S. J.; III, H. F. S., The Gaoh-Hgao Potential Energy Hypersurface and the Necessity of Correlating the 3d Electrons. J. Chem. Phys. 1996, 104, 8516-8523.

33. Austin, A. J.; Frisch, M. J.; Montgomery, J. A.; Petersson, G. A., An Overlap Criterion for Selection of Core Orbitals. Theor. Chem. Acc. 2002, 107, 180-186.

34. Bartlett, R. J.; Musiał, M., Coupled-Cluster Theory in Quantum Chemistry. Rev. Mod. Phys. 2007, 79, 291-352.

35. DeYonker, N. J.; Shah, S. A., The Role of Core-Valence Electron Correlation in Gallium Halides: A Comparison of Composite Methods. Theor. Chem. Acc. 2014, 133, 1518.

36. Minenkov, Y; Bistoni G.; Riplinger, C.; Auer, A. A.; Cavallo, L; Neese, F.; Pair Natural Orbital and Canonical Coupled Cluster Reaction Enthalpies Involving Light to Heavy Alkali and 
Alkaline Earth Metals: The Importance of Sub-Valence Correlation. Phys. Chem. Chem. Phys. 2017, 19, 9373-9391.

37. Figgen, D.; Peterson, K. A.; Dolg, M.; Stoll, H., Energy-Consistent Pseudopotentials and Correlation Consistent Basis Sets for the 5d Elements Hf-Pt. J. Chem. Phys. 2009, 130, 164108.

38. Gong, Y.; Andrews, L.; Bauschlicher, C. W.; Thanthiriwatte, K. S.; Dixon, D. A., Infrared Spectroscopic and Theoretical Studies of the $\mathrm{OTiF}_{2}, \mathrm{OZrF}_{2}$ and $\mathrm{OHfF}_{2}$ Molecules with Terminal Oxo Ligands. Dalton Transactions 2012, 41, 11706-11715.

39. Neese, F., The Orca Program System. Wires Comput. Mol. Sci. 2012, 2, 73-78.

40. van Lenthe, E.; Baerends, E. J.; Snijders, J. G., Relativistic Regular Two-Component Hamiltonians. J. Chem. Phys. 1993, 99, 4597-4610.

41. Weigend, F.; Ahlrichs, R., Balanced Basis Sets of Split Valence, Triple Zeta Valence and Quadruple Zeta Valence Quality for H to Rn: Design and Assessment of Accuracy. Phys. Chem. Chem. Phys. 2005, 7, 3297-3305.

42. Pantazis, D. A.; Neese, F., All-Electron Scalar Relativistic Basis Sets for the Lanthanides. $J$. Chem. Theory. Comput. 2009, 5, 2229-2238.

43. Pantazis, D. A.; Chen, X.-Y.; Landis, C. R.; Neese, F., All-Electron Scalar Relativistic Basis Sets for Third-Row Transition Metal Atoms. J. Chem. Theory. Comput. 2008, 4, 908-919.

44. Peterson, K. A.; Dunning, T. H. Jr. Accurate Correlation Consistent Basis Sets for Molecular Core-Valence Correlation Effects: The Second Row Atoms Al-Ar, and the First Row Atoms B-Ne Revisited. J. Chem. Phys. 2002, 117, 10548-10560.

45. Prascher, B. P.; Woon, D. E.; Peterson, K. A.; Dunning, T. H.; Wilson, A. K., Gaussian Basis Sets for Use in Correlated Molecular Calculations. VII. Valence, Core-Valence, and Scalar Relativistic Basis Sets for Li, Be, Na, and Mg. Theor. Chem. Acc. 2011, 128, 69-82.

46. Koput, J.; Peterson, K. A., Ab Initio Potential Energy Surface and Vibrational-Rotational Energy Levels of $\mathrm{X}^{2} \Sigma^{+} \mathrm{CaOH}$. J. Phys. Chem. A 2002, 106, 9595-9599.

47. DeYonker, N. J.; Peterson, K. A.; Wilson, A. K., Systematically Convergent Correlation Consistent Basis Sets for Molecular Core-Valence Correlation Effects: The Third-Row Atoms Gallium through Krypton. J. Phys. Chem. A 2007, 111, 11383-11393.

48. Balabanov, N. B.; Peterson, K. A., Systematically Convergent Basis Sets for Transition Metals. I. All-Electron Correlation Consistent Basis Sets for the 3d Elements Sc-Zn. J. Chem. Phys. 2005, 123, 064107.

49. Peterson, K. A.; Figgen, D.; Dolg, M.; Stoll, H., Energy-Consistent Relativistic Pseudopotentials and Correlation Consistent Basis Sets for the 4d Elements Y-Pd. J. Chem. Phys. 2007, 126, 124101.

50. Stoychev, G. L.; Auer, A. A.; Neese, F., Automatic Generation of Auxiliary Basis Sets. $J$. Chem. Theory. Comput. 2017, 13, 554-562.

51. Peterson, K. A.; Puzzarini, C., Systematically Convergent Basis Sets for Transition Metals. Ii. Pseudopotential-Based Correlation Consistent Basis Sets for the Group $11(\mathrm{Cu}, \mathrm{Ag}, \mathrm{Au})$ and $12(\mathrm{Zn}$, Cd, Hg) Elements. Theor. Chem. Acc. 2005, 114, 283-296.

52. Bross, D. H.; Peterson, K. A., Correlation Consistent, Douglas-Kroll-Hess Relativistic Basis Sets for the $5 p$ and $6 p$ Elements. Theor. Chem. Acc. 2013, 133, 1434.

53. Boys, S. F.; Bernardi, F. d., The Calculation of Small Molecular Interactions by the Differences of Separate Total Energies. Some Procedures with Reduced Errors. Mol Phys 1970, 19, 553-566.

54. Kendall, R. A.; Dunning Jr, T. H.; Harrison, R. J., Electron Affinities of the First-Row Atoms Revisited. Systematic Basis Sets and Wave Functions. J. Chem. Phys. 1992, 96, 6796-6806.

55. Boys, S. F., Construction of Some Molecular Orbitals to Be Approximately Invariant for Changes from One Molecule to Another. Rev. Mod. Phys. 1960, 32, 296-299. 55. 56 Peterson, K. A.; Yousaf, K. E. Molecular core-valence correlation effects involving the post-d elements $\mathrm{Ga}-\mathrm{Rn}$ : Benchmarks and new pseudopotential-based correlation consistent basis sets. $J$. Chem. Phys. 2010, 133, 174116. 


\section{Graphical TOC entry}

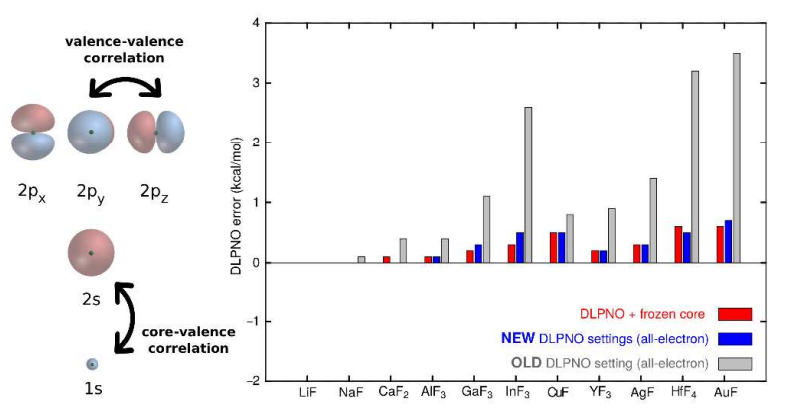

16

17 